
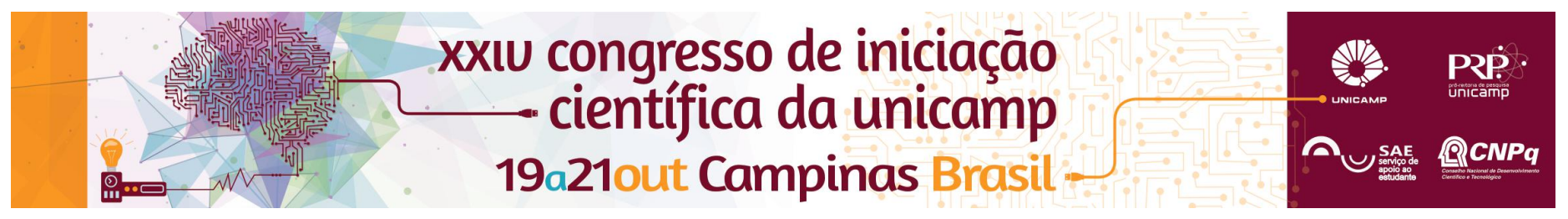

\title{
The construction and evaluation of a hyper-saxophone
}

\author{
Gustavo Nishihara*, Tiago Fernandes Tavares
}

\begin{abstract}
A hyperinstrument is an acoustical musical instrument augmented with attached electronic sensors. These sensors create control signals for sound effect algorithms, leading to new possibilities for musical expression. This project consisted in creating and evaluating a hyper-saxophone. The instrument's sensoring system is centered in a microcontroller, which sends acquired sensor data to a computer. Then, sensor data is used to modulate digital effects. Effect design was part of the research process. The effects were tested in perspective to the musical context chosen for the instrument. Finally, the project proceeded to the creation of a musical repertoire for the new instrument, based in jazz improvisation and live electronics. After this process, we were able to understand the instrument's limitations and plan our next research steps.
\end{abstract}

\section{Key words: \\ New Interfaces for Musical Expression, Arduino, Hyperinstrument}

\section{Introduction}

Hyperinstruments are acoustical musical instruments with attached electronic sensors ${ }^{1}$. They augment the possibilities of musical expression by sonifying the nonmusical actions of the musician.

In this work, we developed a hyper-saxophone using the following methodology. First, we studied and created digital effects using the PureData ${ }^{2}$ music programming environment. After that, we developed a data acquisition device based on Arduino ${ }^{3}$ and electronic sensors. Last, we explored the possibilities brought by the new instrument in musical improvisation sessions, generating an adequate musical repertoire.

The study of digital effects was based on computer music literature ${ }^{4}$. During the study we tested several effects and chose those that produced aesthetically adequate results. In particular, we searched for sound textures that complemented the original sound, instead of substituting it.

The sensoring system is based on an accelerometer and push buttons. The accelerometer was attached to one of the sides of the instrument, capturing the musician's movements. It allows modulating the expression of the digital effects. The push buttons were associated to enabling and disabling effects during a performance. They were attached in a region of easy access to the player, without harming the original instrument's playability.

\section{Results and Discussion}

After the hyperinstrument construction, we started exploring its musical possibilities. For such, we created duet pieces based on jazz and live electronics in musical experimentation sessions. The pieces exploit the expressive possibilities, and add electronic music layers to the hyper-saxophone.

In the current version, the hyper-saxophone still presents some limitations that harm its use. The connection between the microcontroller and the sensors in the instrument is physically fragile, which frequently generates bad connections in the circuit. This can be solved by designing a printed circuit board (PCB) and conceiving a better method to attach sensors to the instrument. Also, the communication between the microcontroller and the computer uses Bluetooth, which has presented intermitent connection problems. Last, the use of PureData makes it necessary to use a support computer, which decreases the instrument portability.

\section{Conclusions}

The hyper-saxophone construction and evaluation was done in 3 parts: the study of digital effects, the development of a sensoring system employing embedded systems techniques, and the exploration of the new possibilities of sound expression by composing, experimental musical pieces.

In future works, we plan to design a PCB, coupling, the microcontroller and the electronic sensors. Also, we will study, using other means of communication in the system like Wi-Fi or ZigBee. Last, we wish to adopt another microcontroller model, with more processing power, to eliminate the need for a support computer.

\section{Acknowledgement}

The author thanks FEEC and NICS, for financial and infrastructural support.

'Tod Machover. Hyperinstruments - a progress report 1987 - 1991. MIT Media Laboratory, 1992.

${ }^{2}$ Puckette, M. S. Pure Data. https://puredata.info/ (acessado em 3 de Julho de 2016).

${ }^{3}$ Arduino. https://www.arduino.cc/ (acessado em 3 de Julho de 2016).

${ }^{4}$ Puckette, M. S. The Theory and Technique of Electronic Music, 2007 